\title{
Valuation of Cryptocurrency Mining Operations
}

\author{
Jose Berengueres $^{\dagger *}$
}

\begin{abstract}
Traditionally, the Net Present Value method has been used to compare diverging investment strategies. However, valuating crypto-projects with fiat-based currency is confusing due to extreme coin appreciation rates as compared to fiat interest rates. Here, we provide a net present value method based on using crypto-coin as the underlying asset. Using this method, we compare buy-and-hold versus mine-and-hold; we also provide a sensitivity analysis of profitability.
\end{abstract}

\section{Introduction}

In the current cryptocurrency mining boom, ${ }^{1,2}$ two opposed views exist on profitability of mining operations. One view states that mining is more profitable, the other states that buying and holding the coin (also known as $\mathrm{HODL}^{3}$ ) is more profitable. ${ }^{4,5}$ However, given any economic criterion, ${ }^{6}$ there is only one optimal strategy. A widely-used criterion to compare investments so far has been Net Present Value (NPV). ${ }^{7,8}$ In the crypto case, we can use it to aggregate the future cash flows that a miner will produce during its lifetime. However, the NPV method is not straightforward to interpret because it depends on the interest rate of the fiat money chosen to measure the cash flow. For example, given a miner that produces coins, assessing its NPV by aggregating future discounted cash flows at a given interest rate is complex because it is not clear what rate should be applied. It is also hard to account or estimate the effect of the appreciation of the coin. Moreover, (unlike fiat) the mined asset does not depreciate - quite the opposite. This poses questions on whether it is appropriate to discount cash flows (coins) that are basically not inflationary. An alternative is to use the Net Coin Value (NCV). The NCV is the sum of the coin flow that a mining operation will produce over $n$ days of mining, minus all expenses valued at the price of the coin on the day of the purchase of the equipment:

$$
\mathrm{NCV}=\sum_{i=1}^{n} C_{i} .
$$

In the above equation, $C_{i}$ is the amount of mined coin at the end of the $i^{\text {th }}$ day minus coins required to pay for the electricity to mine those coins. $C_{i}$ is defined as

$$
C_{i}=\eta \frac{M_{0}}{(1+r)^{i}}-\frac{e}{P_{0}}
$$

†J. Berengueres (jose@uaeu.ac.ae) is Asc. Professor of Computer Science at CIT, UAE University, UAE.

*3QaSKaBqR7h8pUQ8bLWBUrnqpAJW1MVUwE 
where $\eta$ represents the efficiency of the operation after subtraction (in equivalent coin value) of various fees that are approximately proportional or directly proportional to the mining activity (pool fee + mining software fee + hosting, datacenter and admin overheads); $M_{0}$ is the amount of the coin mined on day $0 ; r$ is the daily growth of hashing capacity of all miners mining the coin; and $e$ is the daily electricity bill (in fiat currency) divided by $P_{0}$, the price of the coin on the day the equipment was purchased $(i=0)$. We consider $P_{0}$ to be constant during all the mining period for simplicity and this is a source of error (if the coin value $P$ appreciates the model underestimates the NCV.)

From this, it follows that the payback time happens on the first day of mining that satisfies

$$
\operatorname{NCV}(i)>\frac{\text { cost of miner }}{P_{0}} \text {. }
$$

The time to double the initial investment is then the first day $i$ that satisfies $\operatorname{NCV}(i) \geq$ 2 (cost of miner) $/ P_{0}$.

\section{Valuation Examples}

2.1. Ethereum GPU Mining Case-To illustrate NCV, let us use a real example based on a mining rig composed of $8 \times$ GPU RX580 running the Claymore mining software. In this case, an investor would be interested in finding out whether to invest in the rig or to simply buy and hold coins. Fig. 1 shows a daily cash flow for a scenario where electricity costs $0.19 € / \mathrm{kW}$-hr (Amsterdam rate); the rig costs $\$ 6,756$ of which approximately $\$ 4,000$ is the cost of the GPUs and the rest belongs to power supplies, motherboard, etc. We assume that $\eta$ is $90 \%$. This can vary considerably depending on various fees such as: (i) the admin fee on the mined coins by the pool fees ( $1 \%$ in the case of nanopool.com), (ii) the miner SW fee (1\% in the case of the Claymore miner), and (iii) the data center hosting fee (several dozens of free hosting services exist however usually fees are hidden in the electricity bill, payment terms and small print contract conditions). Four cash flow curves are shown in Fig. 1: the dashed black curve shows the coin value of spending the same amount the rig costs into buying coins and holding them. The solid gray curve is the accumulated coin flow corresponding to buying a rig on day 0 with coins at $P_{0}$ and then accruing the subsequent coins produced. Coin production declines as more mining power is added to the Ethereum network. We use a declining estimation based on exponential growth of the hashing capacity at rate $r$ (Eq. 1). The purple curve shows the $\mathrm{NCV}$ for the same rig, but assuming linear network capacity growth that corresponds to a linear interpolation of the past 12 months provided by Coinwarz, ${ }^{9}$ in this case the equation used is

$$
C_{i}=\eta \frac{M_{0}}{1+i r}-\frac{e}{P_{0}}
$$

In Fig. 1, we assume a $0.45 \%$ daily growth rate (same as the BTC network during some periods in the past, as a fastest-case scenario). Finally, the dashed gray curve shows the daily coin flows using Eq. (1) if the network hash capacity was to grow at the same rate as Moore's Law (modeled as doubling of the hash rate every 18 months) the most optimistic scenario for miners with the slowest growth rate.

As we can see from Fig. 1, the rig recovers the initial investment fast at the beginning and slower later. However, at current estimated network growth rate, it never recoups the cost when we measure value in NCV. Then, about a year since the operation began, the rig will cost 
more to operate than what the mined coins are worth. The accumulated coin produced by the rig never surpasses the buy and hold strategy. Recall that our model assumes that the cost of electricity in coins to be constant, which overestimates the electricity cost measured in coins if the coin appreciates.

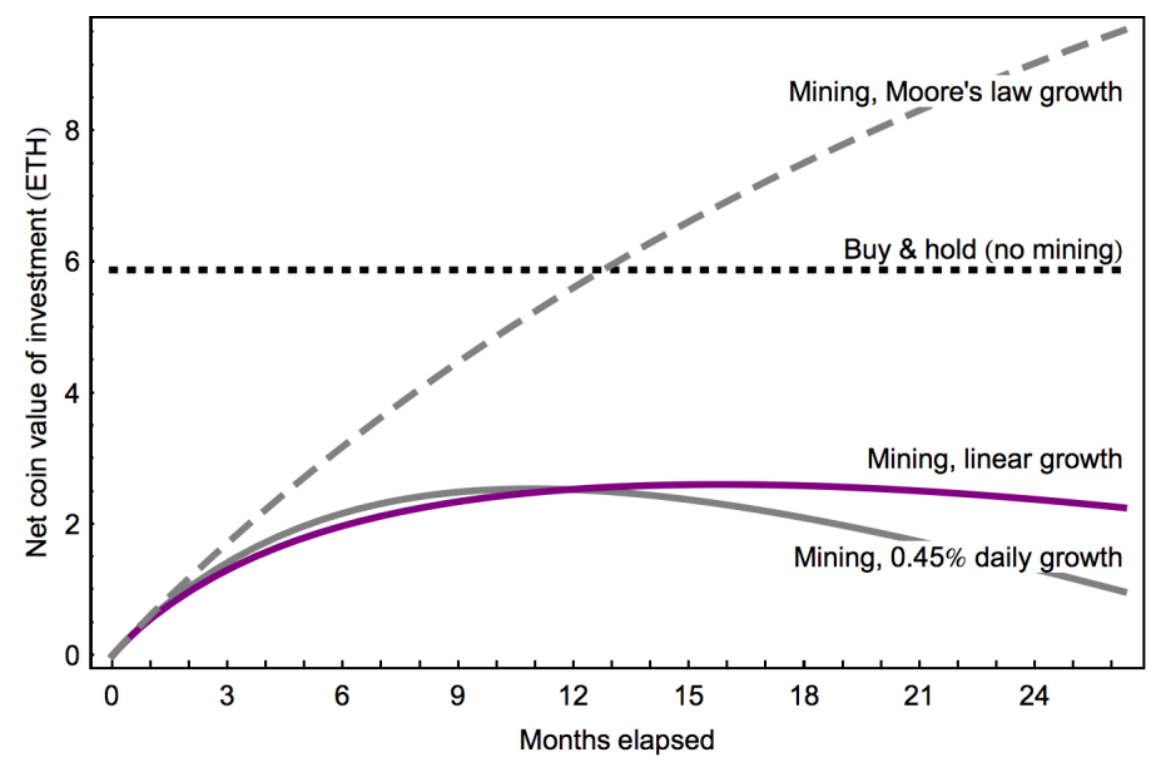

Fig. 1. Accumulated daily cash flow for four investment scenarios at $0.19 €$ per $\mathrm{kW}-\mathrm{hr}$ (Amsterdam prices). At estimated global hashing capacity growth $(0.45 \%$ per day), the max NCV for mining occurs after 1 year and underperforms the buy-and-hold strategy by more than $50 \%$.

2.2. Bitcoin Cash Mining Case-In this example, we will address the profitability of an S9 Miner with parameters as per Table 1. Fig. 2 shows the evolution of NCV measured in Bitcoin Cash $(\mathrm{BCH})$. It compares the NCV for a miner delivered on payment day versus a miner delivered after 4 or 5 months of prepayment. Table 2 summarizes the dramatic effect that delays in delivery of S9 mining machines have on profitability. In Fig. 3 we can also see that the NCV with free electricity provides a hard cap on how much value a rig can produce. We can also see that network growth, rather than electricity cost, is the driving factor impacting the NCV of a mining operation. For example, halving the electricity cost from 0.19 to 0.10 will only increase the (max) NCV from 2.5 to 3.5 coins.

To understand better how changes in different variables impacts NCV we can calculate the Sensitivity Index (SI), ${ }^{10}$ where SI is the ratio between $\left(\mathrm{NCV}_{\mathrm{b}}-\mathrm{NCV}_{1}\right) / \mathrm{NCV}_{\mathrm{b}}$ and $\left(X_{\mathrm{b}}-X_{1}\right) /$ $X_{\mathrm{b}}$. Here, $X_{\mathrm{b}}$ is value of variable in the base case, $X_{1}$ is the value of the variable in the sensitivity test, $\mathrm{NCV}_{\mathrm{b}}$ is the net coin value in the base case and $\mathrm{NCV}_{1}$ is the net coin value in the sensitivity test. 


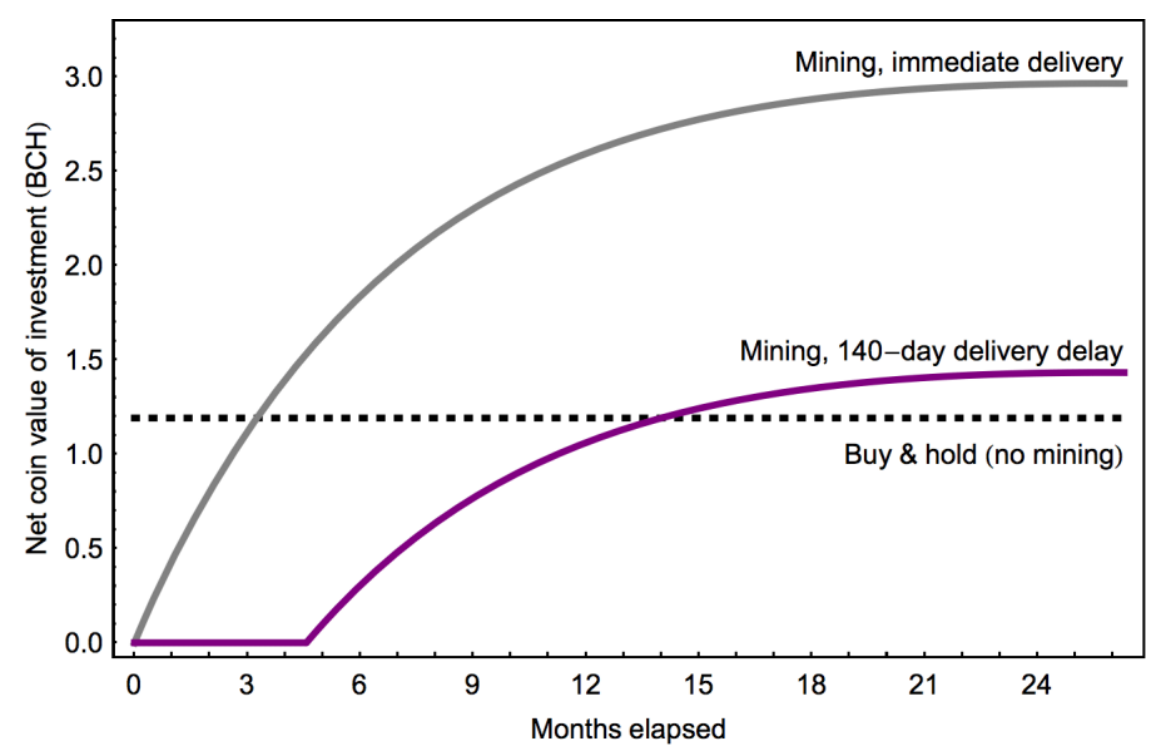

Fig. 2. Prepaying for machines with delays in delivery times has significant impact on profitability at 2016-2017 hashing capacity growth levels.

Table 1. Bitmain Antminer S9 parameters

\begin{tabular}{lr}
\hline Item & value \\
\hline Price S9 in coins & 1.19149 \\
Price S9 in \$ & $\$ 2800.00$ \\
Difficulty increase daily (exponential) & 0.00450 \\
BCH mined per day per miner & 0.01702 \\
Electricity cost in $€$ / $\mathrm{kW}-\mathrm{hr}$ & 0.03000 \\
Electricity cost in BCH / day & 0.00045 \\
$\mathrm{~kW}$ consumed per miner & 1.6 \\
Administration fee & 0.10000 \\
BCH price at miner purchase time & $\$ 2350.00$ \\
Max NCV & $\mathrm{BCH} 2.92$ \\
\hline "Author's estimate
\end{tabular}

Table 2. Effect of Delay on Profitability

\begin{tabular}{lrr}
\hline Item & $\begin{array}{r}\text { No delay in } \\
\text { delivery }\end{array}$ & $\begin{array}{r}140 \text { days } \\
\text { delay }\end{array}$ \\
\hline Max NCV in BCH & 2.925 & 1.253 \\
Max NCV in BCH @ $\mathrm{P}_{0}$ & $\$ 6875$ & $\$ 2946$ \\
Max ROI & 2.45 & 1.05 \\
\hline
\end{tabular}

From Table 3, we can see that NCV is as sensitive to delay in mining, as it is to the cost of equipment. For example, a mere 140 days of delivery delay results in a loss of 1.6 coins, or 
more than half of the potential max NCV, as compared to a machine that starts mining immediately after payment.

Table 3. Sensitivity analysis

\begin{tabular}{|c|c|c|c|c|c|c|c|}
\hline Variable $(X)$ & SI & $X_{\mathrm{b}}$ & $X_{1}$ & $\Delta X / X_{\mathrm{b}}$ & $\mathrm{NCV}_{\mathrm{b}}$ & $\mathrm{NCV}_{1}$ & $\Delta \mathrm{NCV} / \mathrm{NCV}_{\mathrm{b}}$ \\
\hline Network growth rate daily ${ }^{1}$ & $526 \%$ & $0.75 \%$ & $0.18 \%$ & 0.8 & 2 & 10 & -4 \\
\hline Delay in mining in days ${ }^{2}$ & $60 \%$ & 140 & 1 & 1.0 & 3 & 1.2 & 0.6 \\
\hline Electricity cost $€ / \mathrm{kW}-\mathrm{hr}^{2}$ & $54 \%$ & 0.19 & 0.09 & 0.5 & 3.5 & 2.5 & 0.3 \\
\hline Cost of equipment $\$^{2}$ & $41 \%$ & 2800 & 1200 & 0.6 & 2.92 & 3.6 & -0.2 \\
\hline
\end{tabular}

${ }^{1}$ Data from GPU case

${ }^{2}$ Data from table 2

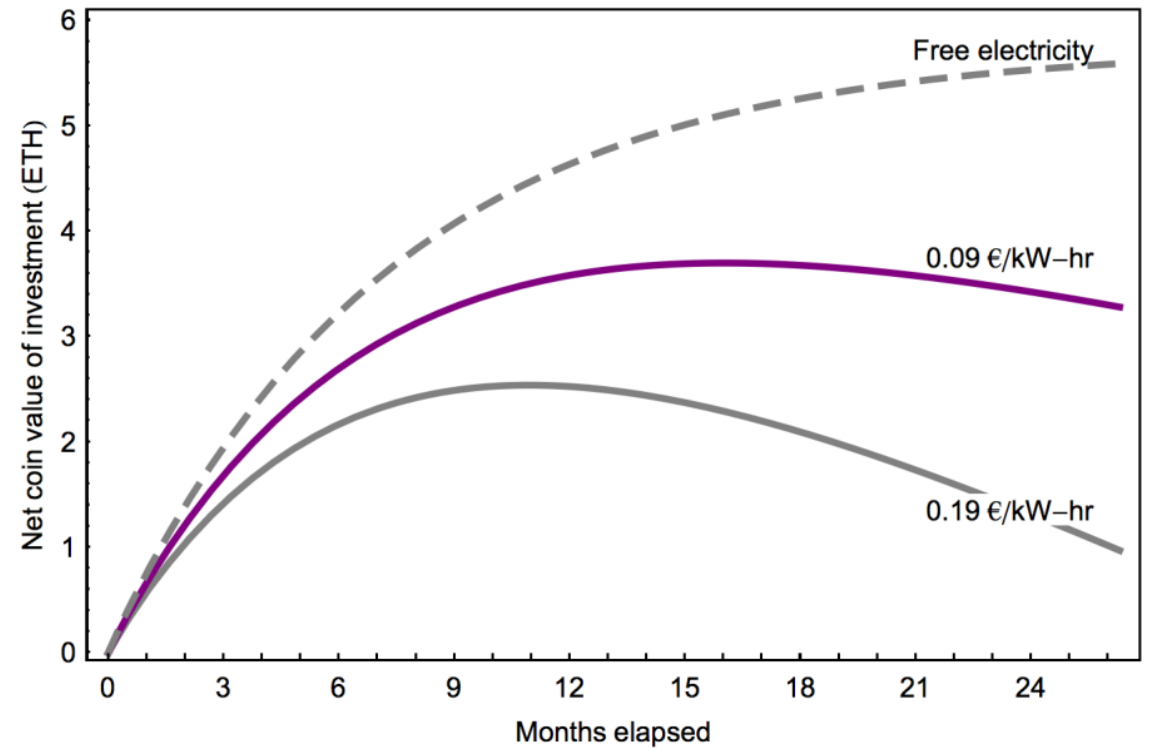

Fig. 3. Effect of different electricity prices on the Net Coin Value the miner. Doubling the electricity price from 0.09 to 0.19 does not halve the NCV proportionately.

2.3. Benefits of Using NCV to Evaluate Projects-Here we will compare NPV to NCV. We show how using NPV can lead to suboptimal investment decisions. Let's assume the case in Fig. 1, a GPU rig to mine Ethereum. Clearly the NCV value of the rig is less than the cost of the machine in coins at the time of purchase (negative ROI). However, if Ethereum was to triple in price since the purchase of the rig (as it happened), and the investor measures the cash flows in USD rather than in ETH he could be fooled into believing that the rig was a good investment decision because the value of the total mined coin after a few months was higher than the cost of the rig in USD, and therefore ROI was positive, not realizing that HODL would have been twice as profitable in fiat terms. Fig. 4 compares the buy-and-hold investment strategy versus a mining-and-hold strategy. The data uses historic price of ETH from Etherscan. Fig. 4 uses NPV, the right chart uses NCV to analyze profitability. 

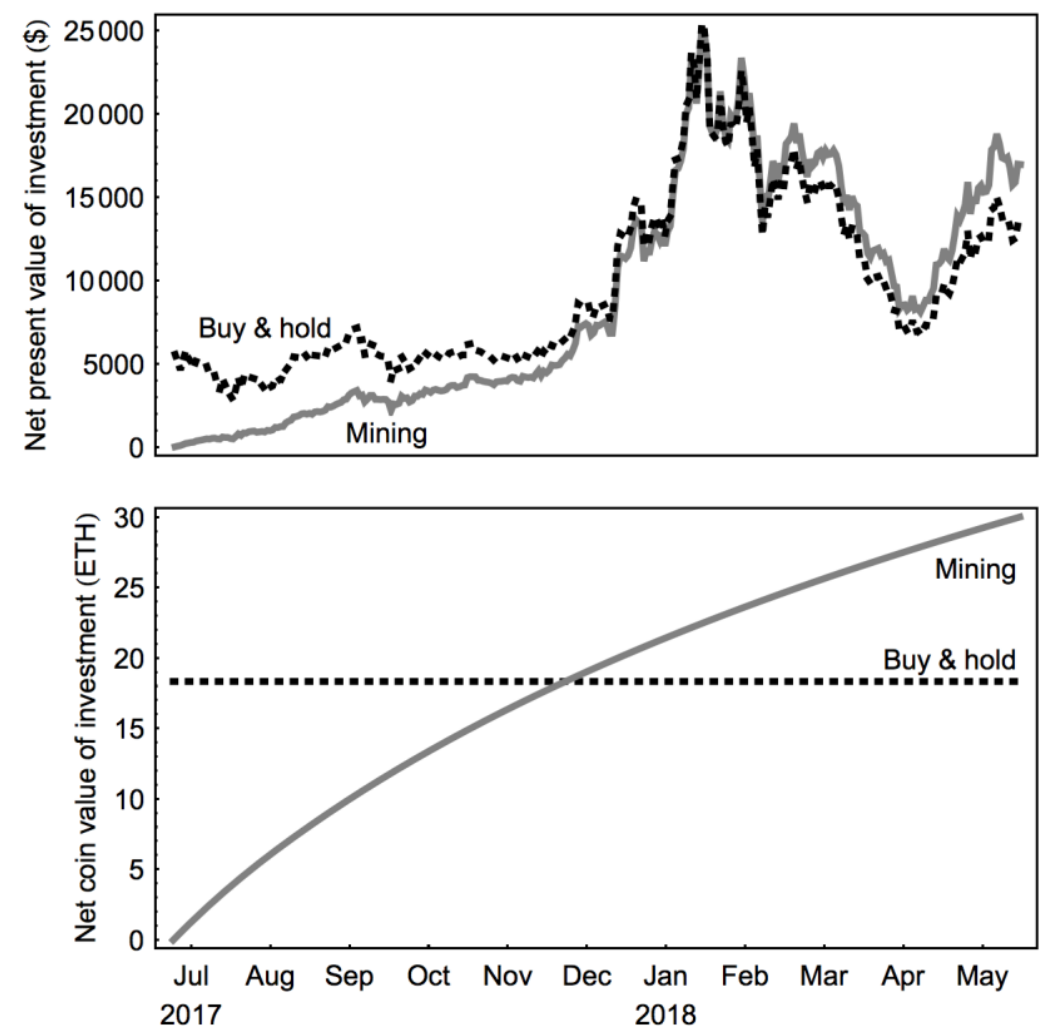

Fig. 4. Top: Net Present Value at a $0 \%$ discount rate is used to compare mining to the buyand-hold investment strategy. Bottom: Net Coin Value is used for same case. The Net Coin Value method is more stable when the coin price volatility is high.

\section{Other factors that impact profitability}

3.1. Cooling Cost-Another factor often overlooked in mining farm projects are the costs of air conditioning (AC), the fire insurance, and so on. For example, in countries, such as Germany, a mining license is required to mine Ethereum even at one's home. In other latitudes, cooling is a significant challenge in hot weather places such as Dubai. In summer, outdoor temperatures can reach up to $55^{\circ} \mathrm{C}$ and cards must not operate at high temperatures. This cost cannot be overlooked in a profitability analysis. Air conditioners and heat pumps have Coefficients of Performance (CoP), typically between 2 to 4, depending on the design and operating conditions. This means that for a $\mathrm{CoP}=2$ for every $2 \mathrm{~kW}$ of GPU heat that we want to dissipate, at least $1 \mathrm{~kW}$ of power must be used by the heat pump or AC just to maintain the temperature inside the mining farm. Therefore, Eq. (1) becomes:

$$
C_{i}=\eta \frac{M_{0}}{(1+r)^{i}}-\left(1+\frac{1}{\mathrm{CoP}}\right) \frac{e}{P_{0}},
$$

Where a CoP value is typically 2 to 4 , and the cost of the AC equipment would be added to rig cost on a pro rata basis.

3.2. Mining With Cards Without Warranty-Overclocking cards increases hashing power between 10 to $20 \%$. For example, from $27 \mathrm{MHz} / \mathrm{s}$ to $32 \mathrm{MHz} / \mathrm{s}$ in the case of an AMD Rx580 
(Ethereum case). This is not a small gain. On the other hand, because mining 24/7 and overclocking abuses the hardware card manufacturers do not issue warranty on so-called mining-cards such as the NVidia p106. Therefore, many farms prefer to use commercial 2year warranty GPU cards such as the NVidia 1060-70-80 consumer series. While the benefits of overclocking with warranty are substantial, overclocking increases the rate of failure of cards but the warranty compensates for that.

3.3. Cycle Lifetime-The NCV peak provides an estimate of when a card becomes unprofitable to operate. Assuming all else constant, we see effective lifetimes of 18 months. Therefore, cost of the card should be treated as an expendable cost, not a capital expenditure in NPV calculations. The 18-month lifetime is surprisingly accurate. For example, today, mining Ethereum with an NVidia GeForce 1060 (launched to market on May 2016, hashing power $10 \mathrm{Mh} / \mathrm{s}$ ), does not produce even $1 / 3 \mathrm{rd}$ of coin produced by an NVidia 1070 card $(27 \mathrm{Mh} / \mathrm{s})$. It produces close to zero due to the way mining pools work (timeouts). If from experience, we consider that the card value for mining drops to zero in 18 months $(n=540$ days) and consider it as a fungible (not a capital expense) then the marginal cost of mining verifies:

$$
C_{i}=\frac{P_{C A R D}}{n},
$$

where $C_{i}$ is given by Eq. (2), $P_{C A R D}$ is the price of the GPU card in coin at purchase time, and $n$ is the number of days mining. Then the number of coin used to compare with the buy-andhold alternative would include all CAPEX in motherboards, PSU, AC and cabling and exclude the cost of GPU cards. Eq. (3) is appropriate because $P_{C A R D}$ is correlated with the price of coins that the card can mine at purchase time while the rest of the equipment is not, and because the lifetime of the rest of the rig is greater than 18 months. From Eq. (3) we can now estimate the marginal pairs (prices of cards, electricity) that make mining marginally profitable. As coin returns diminish, and substituting $i=n$, we can now also forecast if a card will reach its end of lifetime due to obsolescence (Moore's Law boundary) or because a high price of electricity, in which case $n$ should be shortened accordingly in Eq. (3).

\section{Conclusions}

We have shown how to use Net Coin Value method to value mining operations using Ethereum and Bitcoin Cash as the underlying asset. This method offers a simpler alternative to the discounted cash flows method which is not suited for underlying assets that do not depreciate over time. From a qualitative sensitivity analysis, we conclude that there are four main factors that impact profitability in NCV terms. Delivery delay (the time from pre-pay to switch on) has a disproportionate effect on the NCV. Hence, for mining equipment sellers, the easiest way to adjust demand might not be altering the price tag but altering the delivery date on pre-orders. Finally, we can now address the miner's profitability paradox: Mining seems never profitable for new entrants because existing miners that can simply upgrade GPU in their data centers have an unfair capital advantage compared to new entrants, who must invest in the surrounding infrastructure such as cooling, cabling and admin personnel from scratch. We hope this analysis helps to clarify profitability analysis of mining farms. 
LEDGER VOL 3 (2018) 60-67

\section{Acknowledgement}

Thanks to Frank Webber, Beyumi, Ferran Pujol, and Eneko Knorr for fruitful discussion and feedback.

\section{Conflict of Interest}

The author is partner at ai2co.com.

\section{Notes and References}

${ }^{1}$ Kroll, J. A., Davey, I. C., Felten, E. W. "The economics of Bitcoin mining, or Bitcoin in the presence of adversaries." Proceedings of WEIS. 2013 (2013)

https://www.econinfosec.org/archive/weis2013/papers/KrollDaveyFeltenWEIS 2013.pdf.

${ }^{2}$ O’Dwyer, K. J., Malone, D. "Bitcoin mining and its energy footprint." ISSC 2014 / CIICT 2014, Limerick, June 26-27 280-285 (2014)

http://karlodwyer.com/publications/pdf/bitcoin_KJOD_2014.pdf

${ }^{3}$ HODL is an acronym for HOld (the coins) for your Dear Life. It refers to the buy and hold strategy.

${ }^{4}$ Courtois, N. T., Grajek, M., Naik, R. "The unreasonable fundamental incertitudes behind bitcoin mining." arXiv (preprint server) (2013) https://arxiv.org/abs/1310.7935.

${ }^{5}$ Laszka, A., Johnson, B., Grossklags, J. "When bitcoin mining pools run dry.” International Conference on Financial Cryptography and Data Security. Springer, Berlin, Heidelberg, 2015.

${ }^{6}$ Thorp, E. O. "Portfolio choice and the Kelly criterion." In The Kelly Capital Growth Investment Criterion Theory and Practice, World Scientific Publishing Co. 81-90 (2011)

https://EconPapers.repec.org/RePEc:wsi:wschap: 9789814293501 0007.

${ }^{7}$ Ross, S. A. "Uses, abuses, and alternatives to the net-present-value rule." Financial management 24.3 96102 (1995) https://www.jstor.org/stable/3665561.

${ }^{8}$ Remer, D. S., Nieto, A. P. “A compendium and comparison of 25 project evaluation techniques. Part 1: Net present value and rate of return methods." International Journal of Production Economics 42.1 79-96 (1995) https://www.doi.org/10.1016/0925-5273(95)00104-2.

${ }^{9}$ No Author. "Ethereum Network Hashrate Chart and Graph.” CoinWarz.(accessed 15 August 2018) https://www. coinwarz.com/network-hashrate-charts/ethereum-networkhashrate-chart.

${ }^{10}$ Saltelli, A., Chan, K., Scott, E. M., eds. Sensitivity analysis. Vol. 1. New York: Wiley, 2000. 18-19.

(cc) BY

UILIS D-Sore
Articles in this journal are licensed under a Creative Commons Attribution 4.0 License.

Ledger is published by the University Library System of the University of Pittsburgh as part of its D-Scribe Digital Publishing Program and is cosponsored by the University of Pittsburgh Press. 